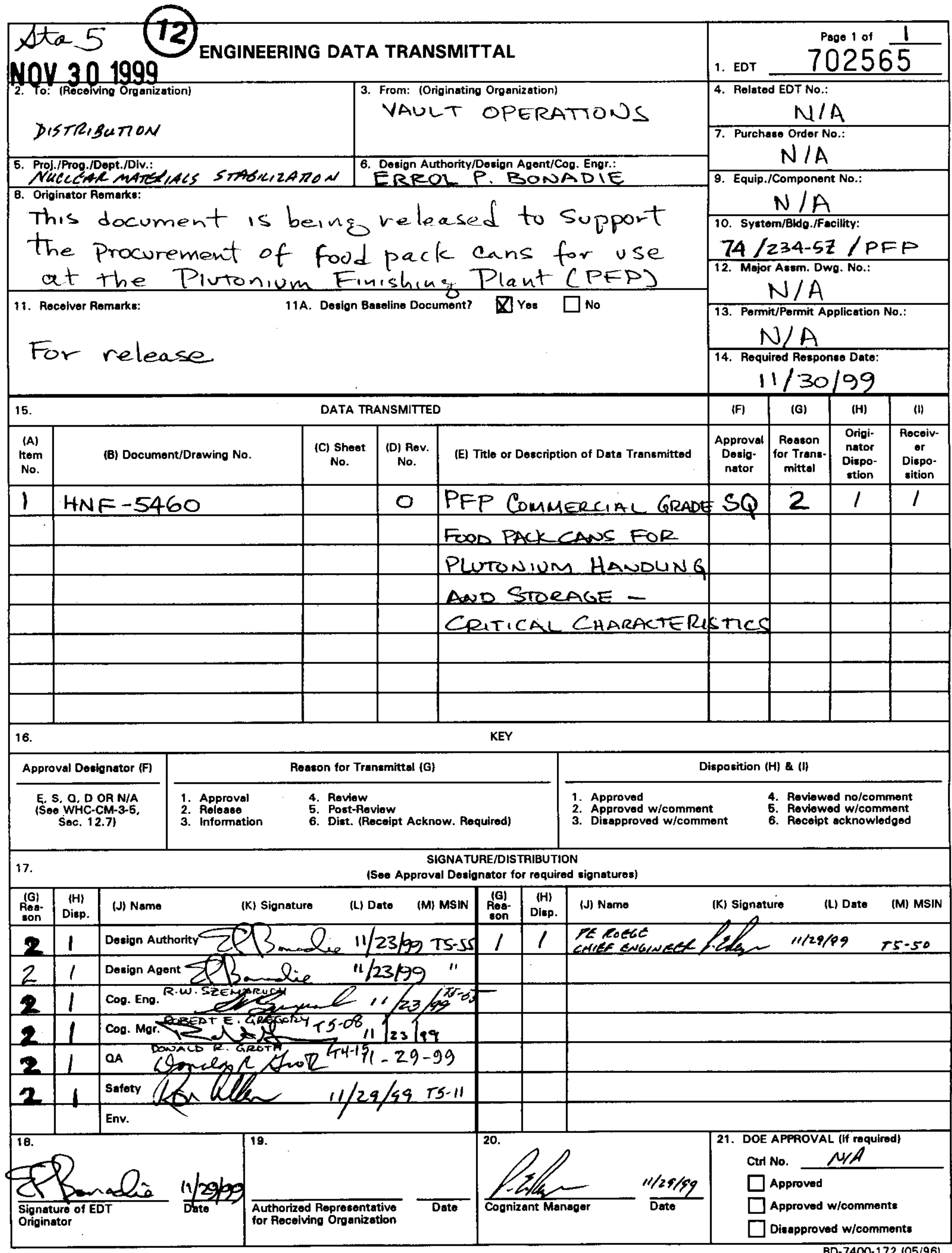

BD-7400-172 (05/96) 


\section{PFP COMMERCIAL GRADE FOOD PACK CANS FOR PLUTONIUM HANDLING AND STORAGE CRITICAL CHARACTERISTICS}

E. P. Bonadie \& R. W. Szempruch

Flour Daniel Hanford Company, Richland, WA 99352

U.S. Department of Energy Contract DE-AC06-96RL13200

EDT/ECN: 702565

Org Code: $15 \mathrm{~V} 00$

B\&R Code: EW04J1050

UC:

Charge Code: 110743

Total Pages: 9

Key Words: PFP, Plate weight, Tin Plate, Commercial Steel, Critical Characteristics

Abstract: This document defines the critical characteristics of Commercial Grade Items procured for use in PFP's Vault Operations.

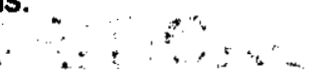

TRADEMARK DISCLAIMER. Referenoe herein to any specific commercial product, process, or senvice by trade name, trademark, menufacturer, or othenvise, does not necesserily constitute or imply its endorsement, recommendation, or favoring by the United States Govemment or any agency thereof or its contractors or subcontractors.

Printed in the United States of America. To obtain copies of this document, contact: Document Control Services, P.O. Box 950, Mailstop H6-08, Richland WA 99352, Phone (509) 372-2420; Fax (509) 376-4989
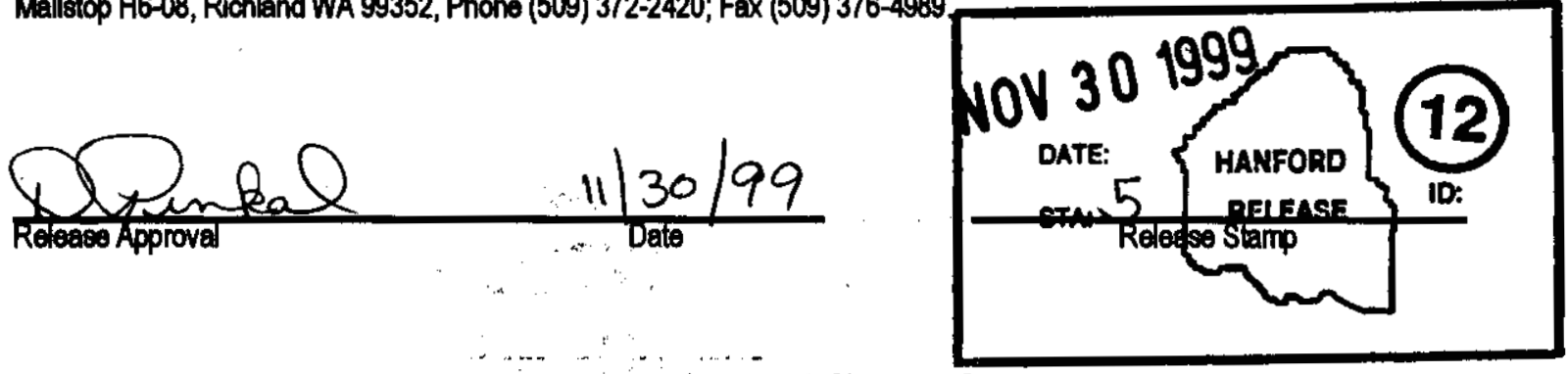

\section{Approved for Public Release}




\subsection{PURPOSE}

This document specifies the critical characteristics for Commercial Grade Items (CGI) procured for PFP's Vault Operations system as required by HNF-PRO-268 and HNF-PRO-1819. These are the minimum specifications that the equipment must meet in order to perform its safety function.

\subsection{BACKGROUND}

PFP's Product Handling and Storage System includes primarily storage containers of the type used in the food industry. These include slip-lid, open top for crimp sealing and various other containers. There are numerous container configurations utilized at the PFP that involve the nesting of several containers, one inside another. Each container in a particular configuration provides one or more safety related functions but typically no two cans provide the same functions. For example, some cans are required to be hermetically sealed while others are not.

Decades of operational experience in storing plutonium at the PFP (Szempruch 1984, Wittman and Bonadie 1996) have demonstrated the acceptability of the use of food industry tin plated steel cans for plutonium storage. Operational requirements for storage of plutonium are controlled by OSD-Z-184-00013. Containers used for plutonium handling and storage are designated Safety Significant in HNF-SD-CP-SAR-021. This Critical Characteristics Document provides a means to document detailed characteristics of food pack containers used at the PFP which previously were specified in individual purchase orders and specifications. The characteristics contained herein are intended to capture documented and undocumented operational experiences, container pressure and drop test results, physical constraints (e.g. storage position size), container monitoring methods, and safety related (e.g. Criticality Prevention Limits on size) requirements of cans used at the PFP. References F and G (Section 6.0), describe results of container drop tests . Federal Specification PPP-C-96E, Cans, Metal, 28 Gage and Lighter is used by agencies of the U.S. Government for the procurement of such cans. Typically, the requirements of PPP-C-96E are easily met by commercial suppliers of tin cans. The Federal Specification is a good source of information to gain an understanding of some of the terminology used within this document.

The procurement requirements associated with containers necessitates procurement of some containers as Commercial Grade Items (CGI) in accordance with HNF-PRO-268, "Control of Purchased Items and Services."

\subsection{SCOPE}

The following list of critical characteristics details specifications for the container configurations currently specified in OSD-Z-184-00013 used for plutonium handling and storage. Material (i.e., metal and electrolytic tin plate thickness) used in the manufacture of the cans should be documented in a letter or Certification of Conformance from the vendor. The other listed characteristics can be measured at FDH receiving. 


\subsection{CRITICAL CHARACTERISTIC LISTING}

\section{307 X 510 (PUREX Slip-Lid Cans)}

Critical Characteristics

- Diameter: 3-7/16 $(-1 / 16)$ inches

- Height: 5-5/8 $( \pm 1 / 32)$ inches

- "Fold-Lock" (double seam) or welded side body seam

- Double seam sealed bottom w/gasket

- $70 \mathrm{lb}$. Plate (minimum): $>0.0077(+0.072 /-0.0005)$ inches

- No. 50 tinned commercial steel or heavier

- Lid: Slip-top

\section{310 X 510 Cans (PFP Slip-Lid)}

Critical Characteristics

- Diameter: $3-5 / 8(-3 / 16)$

- Height: $5-5 / 8(+1 / 32)$ inches

- "Fold-Lock" (double seam) or welded side seam

- Double seam sealed bottom w/gasket

- $70 \mathrm{lb}$. Plate (minimum): $>0.0077(+0.072 /-0.0005)$ inches

- No. 50 tinned commercial steel or heavier

- Lid: Slip-top

\section{401 X 604 (PUREX Secondary Open Top Can)}

Critical Characteristics

- Diameter: $4-1 / 16 \pm 1 / 32$ inches

- Height: 6-1/4 (+1/32 inches)

- Double seam sealed bottom \& lid with gasket

- Welded side seam

- 85 lb. Plate (minimum): 0.0094 (+0.064/-0.0015) inches

- No. 50 tinned commercial steel or heavier 


\section{CRITICAL CHARACTERISTICS}

\section{4. $401 \times 608$ (PFP Secondary Open Top Can)}

Critical Characteristics

- Diameter: $4-1 / 16 \pm 1 / 32$ inches

- Height: 6-1/2 (+1/32 inches)

- Double seam sealed bottom \& lid with gasket

- Welded side seam

- $85 \mathrm{lb}$. Plate (minimum): $0.0094(+0.064 /-0.0015)$ inches

- No. 50 tinned commercial steel or heavier

\section{5. $404 \times 700$ (7-inch Can)}

Critical Characteristics

- Diameter: $4-1 / 4 \pm 1 / 32$ inchés

- Height: $7(+1 / 32)$ inches

- Beaded or paneled wall

- Double seam sealed bottom \& beaded lid with gasket

- $85 \mathrm{lb}$. Plate weight (minimum) 0.0094 (+0.064/-0.0015)

- No. 50 tinned commercial steel or heavier

\section{306 X 800 (Innermost Hanford Convenience Can)}

Critical Characteristics

- No organic material allowed

- Diameter: $3-3 / 8 \pm 1 / 32$ inches

- Height: 8 -inch (+1/32 inches)

- Double seam sealed bottom without gasket

- Welded side seam (no lining stripe)

- $85 \mathrm{lb}$. Plate (minimum): 0.0094 (+0.064/-0.0015) inches

- No. 50 tinned commercial steel or heavier

- Lid: $0.0094(+0.064 /-0.0015)$ inches without gasket

\section{7. $310 \times 804$ (Second Inner Hanford Convenience Can)}

Critical Characteristics

- Diameter: $3-5 / 8 \pm 1 / 32$ inches

- Height: $8-1 / 4(+1 / 32$ inches)

- Double seam sealed bottom \& lid with gasket

- Welded side seam

- $85 \mathrm{lb}$. Plate (minimum): $0.0094(+0.064 /-0.0015)$ inches

- No. 50 tinned commercial steel or heavier 


\section{401 X 900 (Third Hanford Convenience Can)}

Critical Characteristics

- Diameter: $4-1 / 16 \pm 1 / 32$ inches

- Height: 9-inch $(+1 / 32$ inches)

- Double seam sealed bottom \& lid with gasket

- Welded side seam

- $85 \mathrm{lb}$. Plate (minimum): $0.0094(+0.064 /-0.0015)$ inches

- No. 50 tinned commercial steel or heavier

\section{404 X 908 (Outermost Hanford Convenience Can)}

Critical Characteristics

- Diameter: $4-1 / 4 \pm 1 / 32$ inches

- Height: $9-1 / 2(+1 / 32$ inches $)$

- Double seam sealed bottom \& lid with gasket

- Welded side seam

- $85 \mathrm{lb}$. Plate (minimum): 0.0094 ( $+0.064 /-0.0015)$ inches

- No. 50 tinned commercial steel or heavier 


\section{CRITICAL CHARACTERISTICS}

\subsection{APPENDLX}

Container configurations utilized at the PFP involve the nesting of several containers, one inside another. Each container in a particular configuration provides one or more safety related functions, but typically no two cans provide the same functions. For example, some cans are required to be hermetically sealed while others are not. The configurations described below are approved for storing oxides produced from thermal stabilization process. Table 1 describes the packaging configuration using nominal dimensions of each can. Table 2 describes the packaging configuration using the Hanford Convenience Can Packaging dimensions.

Table 1. Thermal Stabilization Product Container Configuration

\begin{tabular}{|c|c|c|c|c|}
\hline $\begin{array}{l}\text { Configuration } \\
\text { Element }\end{array}$ & $\begin{array}{l}\text { "Names" of } \\
\text { Configuration } \\
\text { Element }\end{array}$ & Description & $\begin{array}{l}\text { Nominal } \\
\text { Dimensions } \\
\text { (inches) }\end{array}$ & $\begin{array}{l}\text { Nominal } \\
\text { Volume } \\
\text { (liters) }\end{array}$ \\
\hline Can holding oxide & $\begin{array}{l}\text { PUREX or PFP Slip } \\
\text { Lid Can } \\
(307 \times \mathbf{X 1 0}) \text { or } \\
(\mathbf{3 1 0} \times \mathbf{5 1 0})\end{array}$ & $\begin{array}{l}\text { Tin plated can with } \\
\text { slip lid top } \\
\end{array}$ & $3-7 / 16 \mathrm{D} \times 5-5 / 8 \mathrm{H}$ & 0.8 \\
\hline Plastic Bag & Bagout Bag & $\begin{array}{l}\text { Heat sealed plastic bag } \\
\text { ( }\end{array}$ & $\begin{array}{l}.006 \text { polyethylene or } \\
.012 \mathrm{PVC}\end{array}$ & N/A \\
\hline Secondary Can & $\begin{array}{l}\text { PFP or PUREX } \\
\text { Secondary Can } \\
(401 \times 604) \text { or } \\
(401 \times 608)\end{array}$ & $\begin{array}{l}\text { Tin plated can with } \\
\text { crimp sealed ends }\end{array}$ & $4-1 / 16 \mathrm{D} \times 6-1 / 2 \mathrm{H}$ & 1.3 \\
\hline Outer Can & $\begin{array}{l}7 \text { inch or Juice Can } \\
(404 \times 700)\end{array}$ & $\begin{array}{l}\text { Tin plated can with } \\
\text { crimp sealed ends }\end{array}$ & $4-1 / 4 \mathrm{D} \times 7 \mathrm{H}$ & 1.5 \\
\hline
\end{tabular}

Table 2. Hanford Convenience Container (HCC) Package Configuration

\begin{tabular}{|c|c|c|c|c|}
\hline $\begin{array}{l}\text { Configuration } \\
\text { Element }\end{array}$ & $\begin{array}{l}\text { "Names" of } \\
\text { Configuration } \\
\text { Element }\end{array}$ & 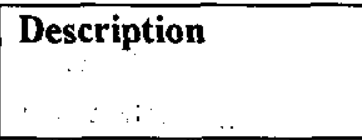 & $\begin{array}{l}\text { Nominal } \\
\text { Dimensions } \\
\text { (inches) } \\
\end{array}$ & $\begin{array}{l}\text { Nominal } \\
\text { Volume } \\
\text { (liters) }\end{array}$ \\
\hline $\begin{array}{l}\text { Material (oxide) } \\
\text { Can }\end{array}$ & $\begin{array}{l}\text { Organic-free Open Top } \\
\text { HCC } \\
(\mathbf{3 0 6} \times \mathbf{8 0 0})\end{array}$ & $\begin{array}{l}\text { Tin plated can with } \\
\text { non-gasketed lids top } \\
\& \text { bottom }\end{array}$ & $3-3 / 8 \mathrm{D} \times 8 \mathrm{H}$ & 1.05 \\
\hline $\begin{array}{l}\text { Can to hold } \\
\text { Material Can }\end{array}$ & $\begin{array}{l}\text { HCC Secondary Can } \\
(310 \text { X 804) }\end{array}$ & $\begin{array}{l}\text { Tin plated can with } \\
\text { elastomeric gaskets } \\
\text { on lids }\end{array}$ & $3-5 / 8 \mathrm{D} \times 8-1 / 4 \mathrm{H}$ & 1.35 \\
\hline Plastic Bag & Bagout Bag & $\begin{array}{l}\text { Heat sealed plastic } \\
\text { bag }\end{array}$ & .006 polyethylene & N/A \\
\hline $\begin{array}{l}\text { First } \\
\text { Contamination- } \\
\text { free Can }\end{array}$ & $\begin{array}{l}\text { HCC Tertiary Can } \\
(401 \times 900)\end{array}$ & $\begin{array}{l}\text { Tin plated can with } \\
\text { crimp sealed ends }\end{array}$ & $4-1 / 16 \mathrm{D} \times 9 \mathrm{H}$ & 1.86 \\
\hline Outermost Can & $\begin{array}{l}\text { HCC Outer Can } \\
(404 \times 908)\end{array}$ & $\begin{array}{l}\text { Tin plated can with } \\
\text { crimp sealed ends }\end{array}$ & $4-1 / 4 \mathrm{D} \times 9-1 / 2 \mathrm{H}$ & 2.15 \\
\hline
\end{tabular}




\subsection{CRITICAL CHARACTERISTIC BASES}

\subsubsection{Slip-Lid Cans}

Can Dimensions: Various and as designated in section 4.0, above.

Basis: required for nesting into next larger can as shown on Table 1 .

Can Wall Thickness: $>70 \#$ plate (>.0077 inches).

Basis: pressure and drop test of "bare" cans. (ref. G).

Can Lid Thickness: >70\# plate ( $>.0077$ inches).

Basis: pressure and drop test of "bare" cans. (ref. G).

Tin Plating Thickness: $>0.50 \#(>.00003$ inch) both sides.

Basis: PFP experience in storage, observations in (Wittman and Bonadie 1996).

Inner can lining: None.

Note: lining stripe over welded seal is not permitted for cans that will be placed into DOE-STD3013-96 compliant containers.

Basis: Storage experience at PFP (Past versions of OSD-Z-184-00013 specified cans intended for storage of wet or damp foods)

Can Closure: Slip-top lid to provide friction surface seal. Can bottom (closed end) either double seam sealed or drawn or spin formed.

Basis: Hermetic sealing not required since this can is nested in other hermetically sealed containers.

Can Side Seams: Welded (preferred) or crimped (folded) seam sealed.

Basis: Certain storage/shipping configurations can result in can temperatures that approach melting point of temperature solders.

Can Wall and Lid Design: Straight wall or beaded design as specified in purchase specification.

Basis: Beaded can walls and lids are desirable from a mechanical strength standpoint.

\subsubsection{Open Top Cans}

Can Type: Type I, Class 3 Packer's Can as defined in PPP-C-96E. Can shall be round, open-top style, with compound-lined, double seamed ends. The material can in the HCC packaging shall not contain any organic compound lining or gaskets.... 
Can Dimensions: Various and as designated in section 4.0, above.

Basis: required for nesting into next larger can as shown on Table 1 and Table 2.

Can Material: Electrolytic tinplate. Basis: Corrosion resistance.

Can Wall Thickness: $>85 \#$ plate (>.0094 inches).

Basis: pressure and drop test of "bare" cans and DOT-6M contents testing

Can Lid Thickness: >85\# plate (>.0094 inches).

Basis: pressure and drop test of "bare" cans (refs. F\& G). Compatibility with pressure monitoring via VSIS and visual inspections. Provides adequate deflection for monitoring while providing adequate margins before failure due to overpressurization.

Tin Plating Thickness: $>0.50$ lbs./base box ( $>.00003$ inch) both sides.

Basis: PFP experience in storage, observations in (Wittman and Bonadie 1996).

Inner can lining: None, except lining stripe over welded seal is permitted. However, the material can in the HCC packaging shall not contain any organic compound lining. Basis: Storage experience at PFP (Past versions of OSD-Z-184-00013 specified cans intended for storage of wet or damp foods). Radiolytic reactions are to be avoided in the HCC.

Can Closure: Hermetic seal by use of industry standard double crimp seal method. Elastomeric gasket on lids and closed end seams (unless closed end has no seam such as drawn or spin formed cans or material can in the HCC packàging).

Basis: Hermetic sealing needed to exclude air/moisture to preserve stabilized oxide product integrity. Provides contamination barrier.

Can Closure Gaskets: Gaskets shall not be broken, cut, torn, chipped or cracked. Gaskets shall not be improperly applied. The material can in the HCC packaging shall not contain organic gaskets.

Basis: Hermetic sealing needed to exclude air/moisture to preserve stabilized oxide product integrity. Provides contamination barrier.

Can Side Seams: Welded in a continuous and uniform way throughout the length of the seam. Basis: Welding assures hermetic seal along seal. Welded seams provide of better lid seam seals at the side seam than crimped seam joints, enhancing ability to obtain hermetic seal. Welding is specified because certain storage/shipping configurations can result in can temperatures that approach melting points of solders.

Can Wall and Lid Design: Straight or beaded wall.

Basis: Nested can arrangement usually requires straight wall cans because of close clearances between cans.

Workmanship: Cans shall be free of lamination, blisters, slivers, rolled-in scales, tears, cracks, cuts, splits, fractures, buckling, dents, wrinkles, or die marks. Plating shall be free of porosity and pits. Basis: PFP experience in storage, observations in (Wittman and Bonadie 1996). 


\subsection{REFERENCES}

A. HNF-PRO-268, "Control of Purchased Items and Services," Rev. 3.

B. HNF-PRO-1819, "PHMC Engineering Requirements," Rev. 3.

C. Federal Specification PPP-C-96E, Cans, Metal, 28 Gage and Lighter, 31-Dec-1992, Defense Supply Center.

D. RHO-HS-SA-59P, The Effectiveness of Corrective Actions Taken to Preclude Events Involving Tin Cans and Plutonium, R. W. Szempruch, May 1984.

E. WHC-SD-CD-TRP-068, Rev 0, Plutonium Inventory Characterization Report 2, G. R. Wittman and E. P. Bonadie, September, 1996.

F. HNF-SD-TP-TRP-002, Rev. 0, Test Report for Hanford Convenience Can, S. R. Crow, January, 1997.

G. ARH-CD-635, Evaluation of Food Pack Cans as Plutonium Storage Containers, J.E. Hammond, March, 1976: 